\title{
Hyperlipidemia and mortality associated with diabetes mellitus co-existence in Chinese peritoneal dialysis patients
}

\author{
Xin Wei ${ }^{1 \dagger}$, Yueqiang Wen ${ }^{2 \dagger}$, Qian Zhou $^{3}$, Xiaoran Feng ${ }^{4}$, Fen Fen Peng ${ }^{5}$, Niansong Wang ${ }^{6}$, Xiaoyang Wang ${ }^{7}$ and \\ Xianfeng $\mathrm{Wu}^{6^{*}}$
}

\begin{abstract}
Background: To evaluate associations between diabetes mellitus (DM) coexisting with hyperlipidemia and mortality in peritoneal dialysis (PD) patients.

Methods: This was a retrospective cohort study with 2939 incident PD patients in China from January 2005 to December 2018. Associations between the DM coexisting with hyperlipidemia and mortality were evaluated using the Cox regression.

Results: Of 2939 patients, with a median age of 50.0 years, 519 (17.7\%) died during the median of 35.1 months. DM coexisting with hyperlipidemia, DM, and hyperlipidemia were associated with 1.93 (95\% Cl 1.45 to 2.56), 1.86 (95\% $\mathrm{Cl} 1.49$ to 2.32), and 0.90 (95\% Cl 0.66 to 1.24)-time higher risk of all-cause mortality, compared with without DM and hyperlipidemia, respectively ( $P$ for trend $<0.001$ ). Subgroup analyses showed a similar pattern. Among DM patients, hyperlipidemia was as a high risk of mortality as non-hyperlipidemia (hazard ratio $1.02,95 \% \mathrm{Cl} 0.73$ to 1.43 ) during the overall follow-up period, but from 48-month follow-up onwards, hyperlipidemia patients had 3.60 ( $95 \% \mathrm{Cl} 1.62$ to 8.01 )-fold higher risk of all-cause mortality than those non-hyperlipidemia ( $P$ interaction $=1.000$ ).

Conclusions: PD patients with DM coexisting with hyperlipidemia were at the highest risk of all-cause mortality, followed by DM patients and hyperlipidemia patients, and hyperlipidemia may have an adverse effect on long-term survival in DM patients.
\end{abstract}

Keywords: Diabetes mellitus, Hyperlipidemia, Mortality, Peritoneal dialysis, Prognosis, Follow-up, Long-term survival

\section{Introduction}

Lipid abnormalities are prevalent in the dialysis population and are influenced by several factors, such as diabetes, renal replacement modalities (hemodialysis and peritoneal dialysis), dietary regimens, and drug use [1,2]. Hemodialysis can moderately reduce renal hyperlipidemia, and peritoneal dialysis patients show more obvious dyslipidemia than

\footnotetext{
*Correspondence: xianfengwu2@163.com

${ }^{+}$Xin Wei and Yuegiang Wen contributed equally to this work.

${ }^{6}$ Department of Nephrology, Affiliated Sixth People's Hospital, Shanghai Jiao Tong University, No.600, Yi Shan Road, Shanghai, China

Full list of author information is available at the end of the article
}

hemodialysis patients, which may be due to the metabolic interference of peritoneal dialysis fluid $[1,3-5]$.

Diabetes mellitus (DM) is the leading cause of endstage renal disease in the United States, accounting for approximately $44 \%$ of incident cases in dialysis settings [6]. DM patients on dialysis have poor survival (34\% over 5 years), worse than those with glomerular disease or hypertension [7]. Compared with dialysis patients without DM, DM patients have a 1.4-fold higher risk of mortality [8]. Hyperlipidemia is prevalent in DM patients on dialysis. Although lipid-modifying medications are associated with a lower risk of cardiovascular events in non- 
dialysis DM patients [9], the association between hyperlipidemia and mortality is inconsistent in DM patients undergoing hemodialysis [9]. A large prospective study has not shown a benefit of statin therapy in reducing cardiovascular events among DM patients on hemodialysis [10]. However, the authors of this study reported that from a 48-month follow-up onwards, patients with lipid-lowering therapy had a better survival rate than those without lipid-lowering therapy using survival curves. Another study reported that in DM patients undergoing hemodialysis, if the low-density lipoprotein before treatment was more than $145 \mathrm{mg} / \mathrm{dl}$, atorvastatin could significantly reduce the risk of death from any cause [11]. A clear association of DM coexisting with hyperlipidemia and mortality can help stratify the risk of death in peritoneal dialysis patients. However, to date, there is no study on these associations. Therefore, in the present study, associations between DM coexisting with hyperlipidemia and mortality were examined in patients on continuous ambulatory peritoneal dialysis (CAPD).

\section{Subjects, materials, and methods Study design and population}

A retrospective cohort study with 3073 incident CAPD patients from five peritoneal dialysis centers in three provinces in China was performed between January 1, 2005, and December 31, 2018. To enhance the generalizability of the findings in the CAPD population, patients younger than 18 years of age or those with less than a 3-month follow-up were excluded. The study was approved by the Human Ethics Committee of each hospital (application ID: [2019]088), consistent with the ethical principles of the Declaration of Helsinki. Written informed consent was obtained from all eligible patients.

\section{Data collection and definitions}

Two trained nurses recorded demographic data, comorbidities, medications, and laboratory parameters at baseline. Two experts evaluated comorbidities by face-to-face interviews and medical records. A total of 2369 (80.6\%) patients were on medication, including calcium channel blockers, beta-blockers, angiotensin II receptor blockers/ angiotensin-converting enzyme inhibitors (ACEI/ARBs), diuretics, statins, and aspirin. Laboratory parameters included hemoglobin, serum albumin, serum uric acid, estimated glomerular filtration rate (eGFR), cholesterol, triglyceride, high-density lipoprotein, low-density lipoprotein, and high-sensitivity C-reactive protein [hs-CRP]).

DM was defined as (1) 2 -h plasma glucose $\geq 200 \mathrm{mg} / \mathrm{dL}$ during an OGTT, (2) HbA1c $\geq 6.5 \%$, (3) fasting plasma glucose $\geq 126 \mathrm{mg} / \mathrm{dL}$, (4) patients with typical symptoms of hyperglycemia, hyperglycemia crisis, or random blood glucose $\geq 200 \mathrm{mg} / \mathrm{dL}$, or (5) using glucose-lowering drugs. In the absence of unequivocal hyperglycemia, criteria 1 to 3 should be confirmed by repeated testing [12]. Hyperlipidemia was defined as (1) serum cholesterol levels $\geq 4.7$ $\mathrm{mmol} / \mathrm{L}$, (2) triglyceride levels $\geq 2.3 \mathrm{mmol} / \mathrm{L}$, or (3) lowdensity lipoprotein levels $\geq 4.1 \mathrm{mmol} / \mathrm{L}$ [13]. Patients who meet one of these three items are defined as having hyperlipidemia. The 169 (8.3\%) patients with pre-existing CVE to prevent recurrence of CVD episodes who were receiving lipid-lowering drugs were not considered to have hyperlipidemia. Hypertension was defined as systolic blood pressure $>140 \mathrm{mmHg}$, diastolic blood pressure $>90$ $\mathrm{mmHg}$ or the use of antihypertensive medications [14]. General hypertension (primary hypertension) was taken into consideration in the present study. CVD was defined as coronary heart disease, congestive heart failure, arrhythmias, cerebrovascular disease, or peripheral vascular disease [15]. Current smoking was defined as at least one cigarette a day, and current alcohol consumption was defined as $>20 \mathrm{~g}$ of ethanol a day [16]. The Chronic Kidney Disease Epidemiology Collaboration equation was used to calculate eGFR [17].

\section{Outcomes and follow-up}

The primary outcome was all-cause mortality. Causes of death were identified by death certificates and medical records. Patients were considered CAPD failures if they died within 3 months from transferring to hemodialysis to death and were not censored.

In light of the new evidence that statin treatment initiation is not recommended in dialysis patients [10, 18, 19], hyperlipidemia patients without lipid-lowering therapy in this study did not receive a new statin treatment when starting CAPD. For clinical purposes (not specifically for this study), all patients needed to return to each center at least quarterly for an overall medical assessment. Eligible patients were followed up until death, CAPD cessation, an 8-year follow-up, or June 30, 2019. Patients who transferred to hemodialysis $\geq 3$ months, received renal transplantation, transferred to other dialysis centers, were lost to follow-up, had an 8-year follow-up, or those after June 30, 2019, were considered to be censored.

\section{Statistical analysis}

The incidence rate was calculated by dividing the proportion of events by the total effective observation time in the risk, which is converted to the number of episodes per 1000 years. Data are shown as the mean \pm standard deviation (SD), median (interquartile range, IQR) or number (\%). Eligible patients were assigned to four groups: group 1 (patients without DM and hyperlipidemia), group 2 (patients with only hyperlipidemia), group 2 (patients with only DM), and group 4 (patients with DM coexisting with hyperlipidemia). Chi-squared, one-way ANOVA, or Kruskal-Wallis tests were used to test for differences in categorical or continuous factor variables among groups. 
Multiple logistic regression analyses were conducted to examine the adjusted odds ratios of DM coexisting with hyperlipidemia, DM, and hyperlipidemia compared with no comorbidities. The factors in the multinomial logistic regression included demographic data, hypertension, preexisting CVD, and laboratory parameters.

Kaplan-Meier curves were used to examine the difference in the cumulative mortality hazard among the four groups over the follow up. Four Cox proportional hazards regression models were conducted to examine the association between DM coexisting with hyperlipidemia and mortality: model 1 , unadjusted; model 2 , model 1 plus demographic and clinical characteristics; model 3, model 2 plus medications; model 4 , model 3 plus laboratory variables. A trend across groups and interactions between age, sex, comorbidities, and all-cause mortality was examined. Statistical analyses were conducted with GraphPad Software 8.0 (GraphPad Prism Software Inc.,
San Diego, California), Stata 15.1. statistical software (StataCorp, College Station, TX), and the $\mathrm{R}$ package 3.6.0 (https://www.r-project.org/), and a $P$ value $<0.05$ was considered significant.

\section{Results}

\section{Baseline characteristics}

Of 3073 potential participants, 42 patients $<18$ years of age were excluded, and 92 with a $<3$-month follow-up were excluded. The remaining 2939 patients were eligible for this study.

Of the 2939 patients with a median age of 50.0 (IQR 39.0-61.0) years, 1697 (57.7\%) were men, 549 (18.7\%) had DM, 533 (18.1\%) had hyperlipidemia, 1915 (65.2\%) had hypertension, and 410 (14.0\%) had pre-existing CVD. In this cohort study, 178 patients $(6.1 \%)$ were assigned to group 4, $371(12.6 \%)$ to group $3,355(12.1 \%)$ to group 2, and $2035(69.2 \%)$ to group 1 . Table 1 shows

Table 1 Baseline demographic characteristics, medications, and laboratory parameters

\begin{tabular}{|c|c|c|c|c|c|}
\hline Variables & No comorbidity & Hyperlipidemia & DM & DM plus hyperlipidemia & $P$-value \\
\hline $\mathrm{N}$ & 2035 & 355 & 371 & 178 & \\
\hline Age $(\mathrm{IQR})$, years & $47.0(37.0-57.0)$ & $50.0(39.0-61.0)$ & $60.0(52.0-67.0)$ & $61.0(52.2-68.0)$ & $<0.001$ \\
\hline Men, \% & $1191(58.5 \%)$ & $176(49.6 \%)$ & $232(62.5 \%)$ & $98(55.1 \%)$ & 0.003 \\
\hline Body mass index (SD), $\mathrm{kg} / \mathrm{m}^{2}$ & $22.2 \pm 5.5$ & $23.5 \pm 14.9$ & $23.4 \pm 3.7$ & $23.9 \pm 3.4$ & $<0.001$ \\
\hline Systolic BP (SD), mmHg & $148.8 \pm 25.8$ & $150.7 \pm 24.7$ & $152.9 \pm 25.6$ & $157.2 \pm 23.2$ & $<0.001$ \\
\hline Diastolic BP (SD), mmHg & $88.6 \pm 16.2$ & $88.9 \pm 14.8$ & $82.1 \pm 13.2$ & $82.9 \pm 14.4$ & $<0.001$ \\
\hline 24-h urine volume (IQR), ml & $800.0(500.0-1200.0)$ & $800.0(425.0-1200.0)$ & $800.0(500.0-1200.0)$ & $800.0(462.5-1287.5)$ & 0.969 \\
\hline Current smoking, (\%) & $192(9.4 \%)$ & $45(12.7 \%)$ & $36(9.7 \%)$ & $21(11.8 \%)$ & 0.239 \\
\hline Current alcohol consumption, (\%) & $73(3.6 \%)$ & $19(5.4 \%)$ & $9(2.4 \%)$ & $7(3.9 \%)$ & 0.209 \\
\hline Hypertension, (\%) & $1210(59.5 \%)$ & $226(63.7 \%)$ & 317 (85.4\%) & $162(91.0 \%)$ & $<0.001$ \\
\hline Pre-existing CVD, (\%) & $169(8.3 \%)$ & $64(18.0 \%)$ & $105(28.3 \%)$ & $72(40.4 \%)$ & $<0.001$ \\
\hline Calcium channel blockers, (\%) & $1446(71.1 \%)$ & $299(84.2 \%)$ & $291(78.4 \%)$ & $165(92.7 \%)$ & $<0.001$ \\
\hline Beta blockers, (\%) & $790(38.8 \%)$ & $187(52.7 \%)$ & $133(35.8 \%)$ & $103(57.9 \%)$ & $<0.001$ \\
\hline Diuretics, (\%) & $111(5.5 \%)$ & $8(2.3 \%)$ & $70(18.9 \%)$ & $11(6.2 \%)$ & $<0.001$ \\
\hline ACEI/ARBs, (\%) & $604(29.7 \%)$ & $156(43.9 \%)$ & $156(42.0 \%)$ & $96(53.9 \%)$ & $<0.001$ \\
\hline Aspirin, (\%) & $109(5.4 \%)$ & $19(5.4 \%)$ & $74(19.9 \%)$ & $42(23.6 \%)$ & $<0.001$ \\
\hline Statins, (\%) & $190(9.3 \%)$ & $83(23.4 \%)$ & $79(21.3 \%)$ & $64(36.0 \%)$ & $<0.001$ \\
\hline Hemoglobin (SD), g/dL & $9.0 \pm 2.8$ & $10.0 \pm 2.7$ & $9.5 \pm 2.9$ & $10.2 \pm 2.5$ & $<0.001$ \\
\hline Serum albumin (SD), g/dL & $3.5 \pm 0.6$ & $3.4 \pm 0.5$ & $3.4 \pm 0.6$ & $3.5 \pm 0.5$ & 0.619 \\
\hline Serum uric acid (SD), mg/dL & $7.0 \pm 2.4$ & $6.9 \pm 2.2$ & $6.6 \pm 2.2$ & $6.9 \pm 2.5$ & 0.076 \\
\hline eGFR (IQR), $\mathrm{mL} / \mathrm{min} / 1.73 \mathrm{~m}^{2}$ & $6.4(4.7-8.2)$ & $6.3(4.7-8.3)$ & $6.7(4.9-8.9)$ & $6.5(4.9-8.2)$ & 0.071 \\
\hline Cholesterol (IQR), mmol/L & $3.9(3.0-4.7)$ & $3.8(3.1 .1-4.7)$ & $3.9(3.1-4.7)$ & $4.1(3.4-4.9)$ & 0.035 \\
\hline Triglyceride (IQR), mmol/L & $1.0(0.6-1.7)$ & $1.0(0.6-1.7)$ & $1.1(0.7-1.8)$ & $1.1(0.7-1.8)$ & 0.923 \\
\hline High-density lipoprotein (IQR), mmol/L & $1.0(0.8-1.3)$ & $1.0(0.8-1.3)$ & $1.0(0.8-1.3)$ & $1.1(0.9-1.3)$ & 0.826 \\
\hline Low-density lipoprotein (IQR), mmol/L & $2.1(1.3-3.0)$ & $2.1(1.3-3.0)$ & $2.1(1.1-3.1)$ & $2.2(1.3-3.3)$ & 0.316 \\
\hline hs-CRP (IQR), mg/L & $4.2(1.9-13.1)$ & $4.5(2.0-17.4)$ & $5.0(2.1-20.8)$ & $4.2(1.9-13.2)$ & 0.068 \\
\hline
\end{tabular}

The variables represented by the mean \pm SD were tested by ANOVA, and the variables represented by IQR were tested by nonparametric tests. DM diabetes mellitus, CVD cardiovascular disease, BP blood pressure, eGFR estimated glomerular filtration rate, $h s$-CRP high-sensitivity C-reactive protein, $S D$ standard deviation, IQR interquartile range 
that compared with group 1 , group 4 was more likely to be elderly, have a higher body mass index, systolic BP, hemoglobin, and cholesterol, have lower diastolic BP levels, be female, have hypertension, have pre-existing CVD, and take medications.

\section{Factors and DM coexisting with hyperlipidemia, DM, and hyperlipidemia}

Table 2 shows that after adjusting for confounding factors, pre-existing CVD, higher body mass index levels, and hemoglobin were independently associated with DM coexisting with hyperlipidemia, DM, and hyperlipidemia using multinomial logistic regression. Additionally, elderly age, hypertension, pre-existing CVD, higher body mass index levels, systolic BP, hemoglobin, and lower levels of diastolic BP were independently associated with a high risk of DM coexisting with hyperlipidemia. Notably, being female was independently associated with hyperlipidemia, and lower levels of serum uric acid were independently associated with DM.

\section{Observational period and mortality}

The overall follow-up period was 10,122.2 patient-years, with a median of 35.1 (IQR 17.9-61.7) months. At the end of the study, 519 patients (17.7\%) had died, including 258 (8.8\%) CVD deaths, 54 (1.8\%) infection deaths, 9 $(0.3 \%)$ gastrointestinal bleeding deaths, $16(0.5 \%)$ tumor deaths, 93 (3.2\%) other death causes, and $82(2.8 \%)$ unknown death causes. Additionally, 353 patients (12.0\%) transferred to hemodialysis, $153(5.2 \%)$ received renal transplants, $26(0.9 \%)$ transferred to other centers, and $100(3.4 \%)$ were lost to follow-up. The incidence rates of all-cause mortality were $142.3,118.2,39.5$, and $36.5 /$
1000 patient-years among groups 4, 3, 2, and 1, respectively.

\section{DM coexisting with hyperlipidemia, DM, and hyperlipidemia and mortality}

Survival analysis found that cumulative survival differed significantly among the four groups (Chi square $=220.3$, $P<0.001$; Fig. 1). Among DM patients from a 48-month follow-up onwards, hyperlipidemia patients had poorer cumulative survival than non-hyperlipidemia patients, suggesting that hyperlipidemia had an adverse effect on the long-term prognosis of DM patients.

Table 3 shows that compared to group 1, groups 4, 3 , and 2 had a 1.93 - (95\% CI 1.45 to 2.56), 1.86- (95\% CI 1.45 to 2.32 ), and 0.90 - (95\% CI 0.66 to 1.24 ) times higher risk of all-cause mortality, respectively, after adjusting for factors in model 4 ( $P$ trend $<0.001)$. The subgroups of men, women, hypertension, nonhypertension, pre-existing CVD, and non-pre-existing CVD showed similar patterns (Fig. 2). There was no interaction among DM coexisting with hyperlipidemia, $\mathrm{DM}$, and hyperlipidemia on all-cause mortality $(P=$ 0.570). $P$ values for the interactions were $>0.05$ for subgroups by sex $(P=0.991)$, hypertension $(P=0.075)$, and pre-existing CVD $(P=0.297)$, suggesting that the increased risk of all-cause mortality associated with DM coexisting with hyperlipidemia, DM, and hyperlipidemia was evident regardless of these factors.

Table 4 shows that compared with hyperlipidemia patients, DM patients had a 2.37 -fold (95\% CI 1.66 to 3.39$)$ higher risk of all-cause mortality in model 4 ( $P$ interaction $=1.000)$. Among DM patients, patients with hyperlipidemia had a similar risk of all-cause mortality compared to those without hyperlipidemia $(H R=1.02$,

Table 2 Associations between variables at baseline and DM coexisting with hyperlipidemia, DM, and hyperlipidemia using the multinomial logistic regression

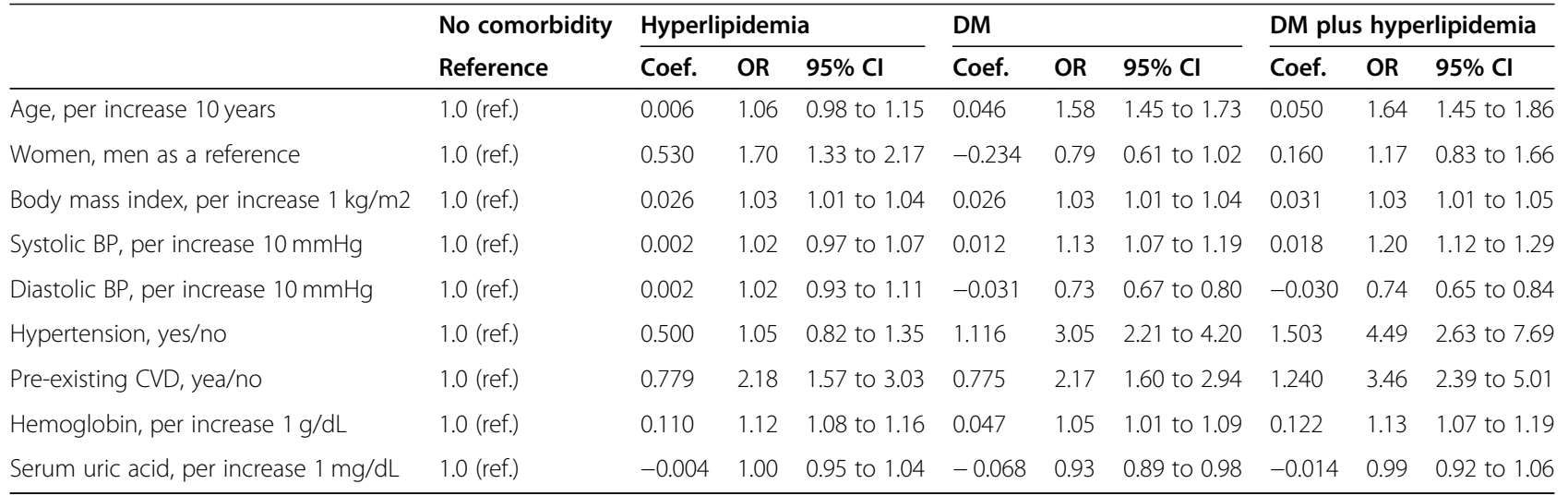

The following variables at baseline were included in the multinomial logistic regression model: age, sex, body mass index, systolic BP, diastolic BP, current smoking, current alcohol consumption, 24-h urine volume, hypertension, pre-existing CVD, hemoglobin, serum albumin, serum uric acid, eGFR, cholesterol, triglyceride, high density lipoprotein, low density lipoprotein, and hs-CRP

$D M$ diabetes mellitus, CVD cardiovascular disease, BP blood pressure, eGFR estimated glomerular filtration rate, $h s$-CRP high-sensitivity C-reactive protein, $O R$ odds ratio, $\mathrm{Cl}$ confidence interval 


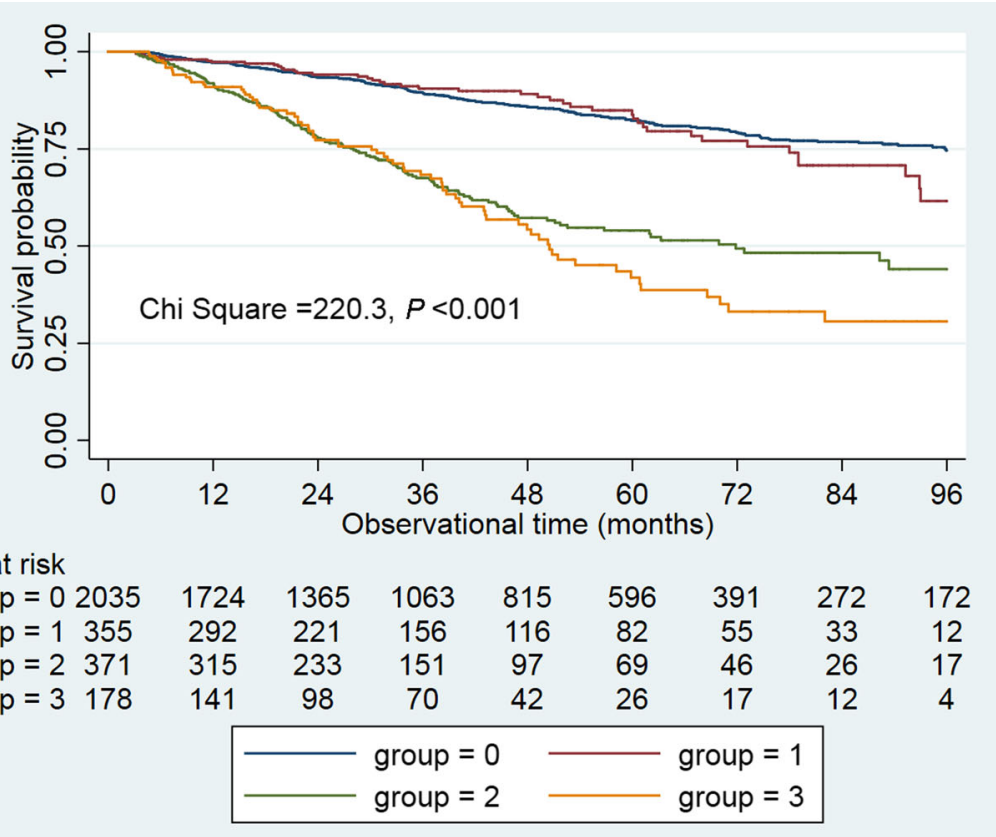

Fig. 1 Cumulative survival curves among four groups. Survival curves showed that from 48-month follow up onwards, DM plus hyperlipidemia group had poor survival than DM group, suggesting among DM patients, hyperlipidemia had a adverse effect on long-term survival. DM, diabetes mellitus

95\% CI 0.73 to 1.43) during the overall follow-up period, but from the 48-month follow-up onwards, patients with hyperlipidemia had a significantly higher risk of all-cause mortality than those without hyperlipidemia $(H R=9.62$, 95\% CI 5.06 to 18.29$)$ after adjusting for confounding factors $(P$ interaction $=1.000)$, which was consistent with the finding of survival curves. Among hyperlipidemia patients, DM patients had a higher risk of all-cause mortality than non-DM patients (HR 2.61, 95\% CI 1.69 to 4.04). In addition, among non-DM patients, hyperlipidemia patients had a similar risk of all-cause mortality (HR 0.97, $95 \%$ CI 0.70 to 1.35 ) as non-hyperlipidemia patients.

\section{Discussion}

In this study, among CAPD patients, baseline DM coexisting with hyperlipidemia was more robustly associated with all-cause mortality than either DM or hyperlipidemia alone. This association was significant regardless of sex, hypertension, and pre-existing CVD. Interestingly, during the overall follow-up period, among DM patients,

Table 3 Adjusted HRs for all-cause mortality among different Cox proportional hazards regression models

\begin{tabular}{|c|c|c|c|c|c|c|c|c|c|c|c|c|c|c|c|c|}
\hline & \multicolumn{4}{|c|}{ Model 1} & \multicolumn{4}{|c|}{ Model 2} & \multicolumn{4}{|c|}{ Model 3} & \multirow[b]{2}{*}{ Coef. } & \multicolumn{3}{|c|}{ Model 4} \\
\hline & Coef. & SE & HR & $95 \% \mathrm{Cl}$ & Coef. & SE & HR & $95 \% \mathrm{Cl}$ & Coef. & SE & $\mathrm{HR}$ & $95 \% \mathrm{Cl}$ & & $\overline{\mathrm{SE}}$ & $\mathrm{HR}$ & $95 \% \mathrm{Cl}$ \\
\hline No comorbidity & & & $\begin{array}{l}1.0 \\
\text { (ref.) }\end{array}$ & & & & & & & & & & & & & \\
\hline Hyperlipidemia & 0.092 & 0.177 & 1.09 & $\begin{array}{l}0.80 \text { to } \\
1.50\end{array}$ & -0.103 & 0.147 & 0.90 & $\begin{array}{l}0.66 \text { to } \\
1.24\end{array}$ & -0.101 & 0.151 & 0.90 & $\begin{array}{l}0.66 \text { to } \\
1.24\end{array}$ & -0.103 & 0.155 & 0.90 & $\begin{array}{l}0.66 \text { to } \\
1.24\end{array}$ \\
\hline $\mathrm{DM}$ & 1.191 & 0.349 & 3.29 & $\begin{array}{l}2.67 \text { to } \\
4.05\end{array}$ & 0.608 & 0.208 & 1.84 & $\begin{array}{l}1.47 \text { to } \\
2.29\end{array}$ & 0.607 & 0.209 & 1.84 & $\begin{array}{l}1.47 \text { to } \\
2.29\end{array}$ & 0.621 & 0.217 & 1.86 & $\begin{array}{l}1.49 \text { to } \\
2.32\end{array}$ \\
\hline $\begin{array}{l}\text { DM plus } \\
\text { hyperlipidemia }\end{array}$ & 1.389 & 0.534 & 4.01 & $\begin{array}{l}3.09 \text { to } \\
5.21\end{array}$ & 0.667 & 0.282 & 1.94 & $\begin{array}{l}1.46 \text { to } \\
2.57\end{array}$ & 0.662 & 0.300 & 1.94 & $\begin{array}{l}1.46 \text { to } \\
2.57\end{array}$ & 0.656 & 0.313 & 1.93 & $\begin{array}{l}1.45 \text { to } \\
2.56\end{array}$ \\
\hline$P$ for trend & & & $\begin{array}{l}< \\
0.001\end{array}$ & & & & $\begin{array}{l}< \\
0.001\end{array}$ & & & & $\begin{array}{l}< \\
0.001\end{array}$ & & & & $\begin{array}{l}< \\
0.001\end{array}$ & \\
\hline
\end{tabular}

Model 1, unadjusted; model 2, model 1 plus age, sex, body mass index, systolic BP, diastolic BP, current smoking, current alcohol consumption, 24-h urine volume, hypertension, and pre-existing CVD; model 3, model 2 plus medications; model 4, model 3 plus hemoglobin, serum albumin, serum uric acid, eGFR, cholesterol, triglyceride, high-density lipoprotein, low-density lipoprotein, and hs-CRP

$D M$ diabetes mellitus, CVD cardiovascular disease, $B P$ blood pressure, eGFR estimated glomerular filtration rate, $h s-C R P$ high-sensitivity C-reactive protein, $S E$ standard error, $H R$ hazard ratio, $C l$ confidence interval 


\begin{tabular}{|c|c|c|c|c|c|}
\hline \multirow{2}{*}{ DM plus hyperlipidemia } & Men & \multicolumn{2}{|c|}{ Hypertension } & \multicolumn{2}{|c|}{ Pre-existing CVD } \\
\hline & $\longmapsto \quad H R 2.2195 \% \mathrm{Cl} 1.65$ to 2.97 & DM plus hyperlipidemia & HR $1.6895 \% \mathrm{Cl} 1.24$ to 2.56 & DM plus hyperlipidemia & $\longmapsto \mathrm{HR} 1.7895 \% \mathrm{Cl} 1.14$ to 2.76 \\
\hline Diabetes mellitus & $\longmapsto \quad H R 1.9095 \%$ Cl 1.24 to 2.91 & Diabetes mellitus & $\longmapsto$ HR $1.5895 \%$ Cl 1.25 to 2.01 & Diabetes mellitus & $\longmapsto$ HR $1.6495 \% \mathrm{Cl} 1.09$ to 2.47 \\
\hline Hyperlipidemia & HR $0.7995 \% \mathrm{Cl} 0.45$ to 1.41 & Hyperlipidemia & — HR $0.9495 \% \mathrm{Cl} 0.65$ to 1.35 & Hyperlipidemia - & 工 HR $0.7995 \% \mathrm{Cl} 0.41$ to 1.53 \\
\hline No comorbidity & ? & No comorbidity & & No comorbidity & \\
\hline \multirow{4}{*}{0} & 3 & 0 & 2 & 0 & 1 \\
\hline & $H R$ & & HR & & HR \\
\hline & Women & & & Non-pr & re-existing CVD \\
\hline & & & Non-hypertension & & \\
\hline DM plus hyperlipidemia & $\longmapsto \quad \mathrm{HR} 1.9495 \% \mathrm{Cl} 1.34$ to 2.82 & DM plus hyperlipidemia & $\longmapsto \mathrm{HR} 6.1795 \% \mathrm{Cl} 2.88$ to 13.22 & DM plus hyperlipidemia & $\longmapsto \mathrm{HR} 1.9595 \% \mathrm{Cl} 1.33$ to 2.84 \\
\hline Diabetes mellitus & $\longmapsto \quad \mathrm{HR} 1.7395 \% \mathrm{Cl} 1.24$ to 2.42 & Diabetes mellitus & - & Diabetes mellitus & $\longmapsto \quad H R 1.8295 \% \mathrm{Cl} 1.40$ to 2.36 \\
\hline Hyperlipidemia & $\longrightarrow \mathrm{HR} 0.9495 \% \mathrm{Cl} 0.63$ to 1.38 & Hyperlipidemia & HR $0.8795 \% \mathrm{Cl} 0.44$ to 1.73 & Hyperlipidemia & H HR $0.9795 \% \mathrm{Cl} 0.67$ to 1.40 \\
\hline No comorbidity & . & No comorbidity & & No comorbidity & \\
\hline 0 & $\frac{1}{2}$ & & $\begin{array}{llllllll}1 & 1 & 1 & 1 & 1 & 1 \\
2 & 4 & 6 & 8 & 10 & 12 & 14\end{array}$ & 0 & $\begin{array}{ll}1 & 1 \\
1 & 2\end{array}$ \\
\hline & HR & & $\mathrm{HR}$ & & HR \\
\hline$P$ intere & action $=0.991$ & $P$ int & raction $=0.075$ & $P$ intera & action $=0.297$ \\
\hline $\begin{array}{l}\text { Fig. } 2 \text { Associatior } \\
\text { the Cox proportic } \\
\text { alcohol consump } \\
\text { cholesterol, trigly } \\
\text { cardiovascular dis } \\
\mathrm{Cl} \text {, confidence int }\end{array}$ & $\begin{array}{l}\text { ns among DM coexisting with } \\
\text { onal hazards regression model } \\
\text { tion, 24-h urine volume, hypert } \\
\text { 'ceride, high-density lipoprotein } \\
\text { sease; BP, blood pressure; eGFR, } \\
\text { terval }\end{array}$ & $\begin{array}{l}\text { yperlipidemia, D } \\
\text { vere included ag } \\
\text { low-dension, pre-existi } \\
\text { estimated glome }\end{array}$ & $\begin{array}{l}\text { 1, and hyperlipidemia and all-cau } \\
\text {, sex, body mass index, systolic } \\
\text { g CVD, medications, hemoglobir } \\
\text { rotein, and hs-CRP, except the s } \\
\text { ular filtration rate; hs-CRP, high-s }\end{array}$ & $\begin{array}{l}\text { use mortality amor } \\
\text { BP, diastolic BP, cu } \\
\text { n, serum albumin, } \\
\text { ubgroup variable. } \\
\text { sensitivity C-reactiv }\end{array}$ & $\begin{array}{l}\text { urrent smokgroups. Covariables in } \\
\text { serum uric acid, eGFR, } \\
\text { DM, diabetes mellitus; CVD, } \\
\text { ive protein; HR, hazards ratio; }\end{array}$ \\
\hline
\end{tabular}

Table 4 Association among DM and hyperlipidemia and all-cause mortality*

\begin{tabular}{|c|c|c|c|c|c|c|c|c|c|c|c|c|c|c|c|c|}
\hline & \multicolumn{4}{|c|}{ Model 1} & \multicolumn{4}{|c|}{ Model 2} & \multicolumn{4}{|c|}{ Model 3} & \multicolumn{4}{|c|}{ Model 4} \\
\hline & Coef. & SE & HR & $95 \% \mathrm{Cl}$ & $\overline{\text { Coef. }}$ & SE & HR & $95 \% \mathrm{Cl}$ & Coef. & SE & HR & $95 \% \mathrm{Cl}$ & Coef. & SE & HR & $95 \% \mathrm{Cl}$ \\
\hline Hyperlipidemia group & & & $\begin{array}{l}1.0 \\
\text { (ref.) }\end{array}$ & & & & & & & & & & & & & \\
\hline DM group & 1.090 & 0.319 & 3.01 & $\begin{array}{l}2.14 \text { to } \\
4.21\end{array}$ & 0.811 & 0.238 & 2.24 & $\begin{array}{l}1.58 \text { to } \\
3.18\end{array}$ & 0.811 & 0.238 & 2.24 & $\begin{array}{l}1.58 \text { to } \\
3.18\end{array}$ & 0.858 & 0.251 & 2.37 & $\begin{array}{l}1.66 \text { to } \\
3.39\end{array}$ \\
\hline
\end{tabular}

Among DM patients

The follow-up period

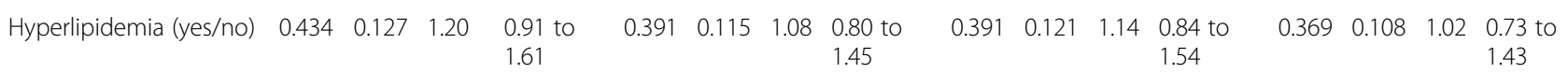

From 48-moth follow

up onwards

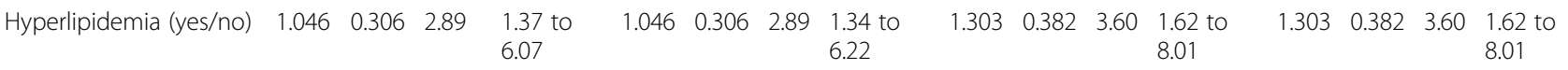

Among non-DM

patients

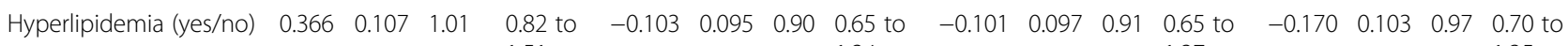

$\begin{array}{lllll}1.51 & 1.24 & 1.27 & 1.35\end{array}$

Among hyperlipidemia

patients

\begin{tabular}{|c|c|c|c|c|c|c|c|c|c|c|c|c|c|c|c|c|c|c|}
\hline DM (yes/no) & 1.368 & 0.401 & 3.78 & $\begin{array}{l}2.60 t \\
5.50\end{array}$ & 0.94 & 0.2 & 32. & & $1.74 \mathrm{tc}$ & 1.0 & & 0.30 & 2.8 & & & & & \\
\hline
\end{tabular}

${ }^{*} P$ interaction $=1.000$

Model 1, unadjusted; model 2, model 1 plus age, sex, body mass index, systolic BP, diastolic BP, current smoking, current alcohol consumption, 24-h urine volume, hypertension, and pre-existing CVD; model 3, model 2 plus medications; model 4, model 3 plus hemoglobin, serum albumin, serum uric acid, eGFR, cholesterol, triglyceride, high-density lipoprotein, low-density lipoprotein, and hs-CRP

$D M$ diabetes mellitus, CVD cardiovascular disease, BP blood pressure, eGFR estimated glomerular filtration rate, $h s-C R P$ high-sensitivity C-reactive protein, $S E$ standard error, $H R$ hazard ratio, $\mathrm{Cl}$ confidence interval 
hyperlipidemia patients were at a similarly high risk of all-cause mortality as non-hyperlipidemia patients. However, from the 48-month follow-up onwards, hyperlipidemia patients had a 3.60-fold higher risk of all-cause mortality than non-hyperlipidemia patients, suggesting that hyperlipidemia had an adverse effect on the longterm survival of DM patients. Among non-DM patients, hyperlipidemia did not affect mortality irrespective of the follow-up duration. In addition, DM patients had a 2.37 -fold higher risk of all-cause mortality than hyperlipidemia patients.

Studies in the general population have shown that reducing total cholesterol and low-density lipoprotein levels can significantly reduce cardiovascular events and mortality [20-22]. However, in dialysis patients, several epidemiological investigations and randomized clinical trials have failed to show the beneficial effect of lipid-lowering therapy in reducing CVD, although low-density cholesterol levels are significantly reduced $[10,18,19,23]$. Dyslipidemia is commonly observed in dialysis patients and is characterized by abnormal composition and concentrations of plasma lipoproteins [24]. People are paying increasing attention to the harmful effects of glucose-based PD solutions on various metabolic changes (such as lipid abnormalities) [25]. In fact, several studies have shown that compared with hemodialysis patients, the lipid distribution of PD patients has stronger atherosclerosis [26-29]. The serum total cholesterol and low-density cholesterol levels of PD patients are usually elevated, while the serum levels of cholesterol and low-density cholesterol levels in hemodialysis patients are normal or low. In addition, PD patients' serum triglyceride levels are much higher than those of hemodialysis patients, and the serum high-density cholesterol levels of both patient populations are usually low [26-29]. Elevated nonhigh-density lipoprotein cholesterol can predict atherosclerotic cardiovascular events in hemodialysis patients [30].

This was an observational cohort study from a nationwide dialysis registry in Japan of 45,390 hemodialysis patients without pre-existing CVD with 44,190 personyears. Higher cholesterol and high-density cholesterol levels in hemodialysis patients were associated with a lower risk of all-cause mortality. A previous study examined the relationship between time-varying serum lipid concentration and all-cause and CVD mortality in a 10year follow-up of $749 \mathrm{PD}$ patients. In contrast to the general population, in PD patients, lower cholesterol and low-density cholesterol levels over time were significantly associated with adverse events and CVD mortality. Although lower triglyceride and high-density lipoprotein cholesterol concentrations were associated with a significantly higher risk of all-cause mortality, they failed to show any significant association with CVD mortality [31]. In the present study, we defined hyperlipidemia according to lipid management in the Chinese population. Among CAPD patients, DM coexisting with hyperlipidemia at baseline was more strongly associated with all-cause mortality than DM or hyperlipidemia alone. Notably, hyperlipidemia harmed long-term survival in DM patients but had no effect in non-DM patients. In addition, DM was independently associated with a higher risk of all-cause mortality in hyperlipidemia patients. In this study, the diagnosis of hyperlipidemia was based on the 2016 Chinese Guideline for the Management of Hyperlipidemia in Adults, which only included higher lipid profiles. Thus, patients with lower lipid profiles may be assigned to the non-hyperlipidemia group; lower lipid profiles are prevalent and associated with a higher risk of mortality in dialysis [32-34]. This study's assignment may affect the association among DM coexisting with hyperlipidemia, DM, and hyperlipidemia and mortality. Additionally, in this study, there was no significant difference in lipid profiles between patients with hyperlipidemia and those without, with the exception of cholesterol. This may be because a patient with high cholesterol may have low triglycerides or low-density lipoprotein. In the future, clinical trials with PD patients should consider hyperlipidemia as a primary or secondary endpoint.

The results of subgroup analyses highlighted the consistency in the association between DM coexisting with hyperlipidemia and the risk of all-cause mortality across men and women, hypertension and non-hypertension, and pre-existing CVD and non-pre-existing CVD. However, it is noteworthy that the strength of the association between DM coexisting with hyperlipidemia and the risk of all-cause mortality may vary by sex, hypertension, and pre-existing CVD. Interestingly, among DM patients, patients with hyperlipidemia had a similar risk of mortality as those without hyperlipidemia during the overall follow-up period. However, among DM patients, from the 48-month followup onwards, patients with hyperlipidemia had a 3.60 -fold higher risk of mortality than those without hyperlipidemia. Statins accounted for $36.0 \%$ of patients with DM coexisting with hyperlipidemia and $9.3 \%$ in their counterparts. The lower percentiles of statin use were due to the fact that statin treatment initiation is no longer recommended in dialysis patients $[10,18,19]$.

\section{Study strengths and limitations}

The study's strengths were its sizeable multicenter sample size, the generalized inclusion and exclusion criteria of the sample, and rigorous multivariate regression analyses.

This study had some limitations. First, this was a retrospective cohort study involving potential unexplained confounding factors and selection bias. Even after adjusting for confounding variables at baseline, we did not reach a conclusion about the potential causality between comorbidities and mortality. Second, defining hyperlipidemia was challenging. In this study, given the 
effect of ethnicity and district characteristics on the Chinese CAPD population, the diagnosis of hyperlipidemia was based on the 2016 Chinese Guideline for Managing Hyperlipidemia in Adults [13], which only included higher lipid profiles. The definition may underestimate the prevalence of dyslipidemia. Third, because dynamic changes in serum lipids were not recorded, the effect of the serum lipid profile on mortality was not examined. Nonetheless, if patients had significant serum lipid disorders during the follow-up period, an integrated approach including lifestyle modifications, nutritional supplements, anti-inflammatory drugs, and improved dialysis therapy to improve serum lipid disorders was conducted [35]. Finally, to strengthen the generalizability of the findings in the CAPD population, patients younger than 18 years of age or patients with a follow-up period of less than 3 months were excluded. Furthermore, all eligible patients were from China, suggesting that the findings may lack generalizability to other ethnic population settings.

\section{Conclusion}

This study showed that among CAPD patients, patients with DM coexisting with hyperlipidemia at the start of CAPD were at the highest risk of mortality, followed by DM patients and hyperlipidemia patients, and DM patients had a higher risk of mortality than hyperlipidemia patients. In addition, among hyperlipidemia patients, DM was independently associated with a high risk of mortality. It is worth noting that hyperlipidemia may harm long-term survival in DM patients but has no effect in non-DM patients. The findings suggest that clear associations between DM coexisting with hyperlipidemia, DM, and hyperlipidemia and mortality may further help with the stratification of the risk of mortality in CAPD patients with comorbidities.

\section{Abbreviations}

CAPD: continuous ambulatory peritoneal dialysis; DM: diabetes mellitus; CVD: cardiovascular disease; BP: blood pressure; eGFR: estimated glomerular filtration rate; hs-CRP: high-sensitivity C-reactive protein; SD: standard deviation; IQR: interquartile range; OR: odds ratio; HR: hazard ratio; Cl: confidence interval

\section{Acknowledgements}

Thank all patients who participated in the study.

\section{Authors' contributions}

All authors contributed to the study conception and design. Material preparation, data collection and analysis were performed by all authors. The first draft of the manuscript was written by XW and XF, and all authors commented on previous versions of the manuscript. All authors read and approved the final manuscript.

\section{Funding}

This research did not receive any specific grant from funding agencies in the public, commercial, or not-for-profit sectors.

\section{Availability of data and materials}

The datasets used and/or analyzed during the current study are available from the corresponding author on reasonable request.

\section{Ethics approval and consent to participate}

The study was approved by the Human Ethics Committee of each hospital (application ID: [2019]088), consistent with the Declaration of Helsinki's ethical principles. Written informed consent was obtained from all eligible patients.

\section{Consent for publication}

Written informed consent for publication of their clinical details was obtained from the patient.

\section{Competing interests}

The authors declare that they have no competing interests.

\section{Author details}

${ }^{1}$ Department of Nephrology, the First Affiliated Hospital of Nanchang University, Nanchang, China. ${ }^{2}$ Department of Nephrology, the Second Affiliated Hospital of Guangzhou Medical University, Guangzhou, China. ${ }^{3}$ Department of Medical Statistics, Clinical Trials Unit, The First Affiliated Hospital, Sun Yat-sen University, Guangzhou, China. ${ }^{4}$ Department of Nephrology, Jiujiang No. 1 People's Hospital, Jiujiang, China. ${ }^{5}$ Department of Nephrology, Zhujiang Hospital of Southern Medical University, Guangzhou, China. ${ }^{6}$ Department of Nephrology, Affiliated Sixth People's Hospital, Shanghai Jiao Tong University, No.600, Yi Shan Road, Shanghai, China. ${ }^{7}$ Department of Nephrology, The First Affiliated Hospital of Zhengzhou University, Zhengzhou, China.

Received: 16 August 2020 Accepted: 19 October 2020

Published online: 07 November 2020

\section{References}

1. Moradi H, Vaziri ND. Molecular mechanisms of disorders of lipid metabolism in chronic kidney disease. Front Biosci (Landmark Ed). 2018;23:146-61.

2. Wanner C, Tonelli M, Kidney Disease: Improving Global Outcomes Lipid Guideline Development Work Group M. KDIGO clinical practice guideline for lipid management in CKD: summary of recommendation statements and clinical approach to the patient. Kidney Int. 2014;85:1303-9.

3. Attman PO, Samuelsson O, Johansson AC, Moberly JB, Alaupovic P. Dialysis modalities and dyslipidemia. Kidney Int Suppl. 2003;84:S110-2.

4. Vaziri ND. Disorders of lipid metabolism in nephrotic syndrome: mechanisms and consequences. Kidney Int. 2016;90:41-52.

5. Clementi A, Kim JC, Floris M, Cruz DN, Garzotto F, Zanella M, Nalesso F, Brendolan A, Giavarina D, Soffiati G, Ronco C. Statin therapy is associated with decreased small, dense low-density lipoprotein levels in patients undergoing peritoneal dialysis. Contrib Nephrol. 2012;178:111-5.

6. Collins AJ, Foley RN, Chavers B, Gilbertson D, Herzog C, Johansen K, Kasiske B, Kutner N, Liu J, St Peter W, et al. 'United States Renal Data System 2011 Annual Data Report: Atlas of chronic kidney disease \& end-stage renal disease in the United States. Am J Kidney Dis. 2012;59:A7, e1-420.

7. Rhee CM, Leung AM, Kovesdy CP, Lynch KE, Brent GA, Kalantar-Zadeh K Updates on the management of diabetes in dialysis patients. Semin Dial. 2014:27:135-45.

8. Foley RN, Murray AM, Li S, Herzog CA, McBean AM, Eggers PW, Collins AJ. Chronic kidney disease and the risk for cardiovascular disease, renal replacement, and death in the United States Medicare population, 1998 to 1999. J Am Soc Nephrol. 2005;16:489-95.

9. Chen SC, Tseng CH. Dyslipidemia, kidney disease, and cardiovascular disease in diabetic patients. Rev Diabet Stud. 2013;10:88-100.

10. Wanner C, Krane V, Marz W, Olschewski M, Mann JF, Ruf G, Ritz E, German D, Dialysis Study I. Atorvastatin in patients with type 2 diabetes mellitus undergoing hemodialysis. N Engl J Med. 2005;353:238-48.

11. Marz W, Genser B, Drechsler C, Krane V, Grammer TB, Ritz E, Stojakovic T, Scharnagl H, Winkler K, Holme I, et al. Atorvastatin and low-density lipoprotein cholesterol in type 2 diabetes mellitus patients on hemodialysis. Clin J Am Soc Nephrol. 2011;6:1316-25.

12. American Diabetes A. Diagnosis and classification of diabetes mellitus. Diabetes Care. 2011;34(Suppl 1):S62-9.

13. Joint committee issued Chinese guideline for the management of dyslipidemia in a. 2016 Chinese guideline for the management of dyslipidemia in adults. Zhonghua Xin Xue Guan Bing Za Zhi. 2016;44: 833-53. 
14. Branch CMDAN. 2016 guidelines for the management of renal hypertension in China. Chin Med J. 2017;97:9.

15. Wen Y, Zhan X, Wang N, Peng F, Feng X, Wu X. Monocyte/lymphocyte ratio and cardiovascular disease mortality in peritoneal Dialysis patients. Mediat Inflamm. 2020;2020:9852507.

16. Tu W, Wu J, Jian G, Lori J, Tang Y, Cheng H, Wu X, Wang N. Asymptomatic hyperuricemia and incident stroke in elderly Chinese patients without comorbidities. Eur J Clin Nutr. 2019;73:1392-402.

17. Zhang L, Wang F, Wang L, Wang W, Liu B, Liu J, Chen M, He Q, Liao Y, Yu X, et al. Prevalence of chronic kidney disease in China: a cross-sectional survey. Lancet. 2012;379:815-22.

18. Fellstrom BC, Jardine AG, Schmieder RE, Holdaas H, Bannister K, Beutler J, Chae DW, Chevaile A, Cobbe SM, Gronhagen-Riska C, et al. Rosuvastatin and cardiovascular events in patients undergoing hemodialysis. N Engl J Med. 2009:360:1395-407.

19. Baigent C, Landray MJ, Reith C, Emberson J, Wheeler DC, Tomson C, Wanner C, Krane V, Cass A, Craig J, et al. The effects of lowering LDL cholesterol with simvastatin plus ezetimibe in patients with chronic kidney disease (Study of heart and renal protection): a randomised placebocontrolled trial. Lancet. 2011;377:2181-92.

20. LaRosa JC, Grundy SM, Waters DD, Shear C, Barter P, Fruchart JC, Gotto AM, Greten H, Kastelein JJ, Shepherd J, et al. Intensive lipid lowering with atorvastatin in patients with stable coronary disease. N Engl J Med. 2005; 352:1425-35.

21. Heart Protection Study Collaborative G. MRC/BHF heart protection Study of cholesterol lowering with simvastatin in 20,536 high-risk individuals: a randomised placebo-controlled trial. Lancet. 2002;360:7-22.

22. Swieczkowska A, Konieczny W, Jasinski W, Zakrzewski K. Interlaboratory comparison of the results of radioimmunoassays. Pol Przegl Radiol. 1985;49:271-6.

23. Bowden RG, La Bounty P, Shelmadine B, Beaujean AA, Wilson RL, Hebert S Reverse epidemiology of lipid-death associations in a cohort of end-stage renal disease patients. Nephron Clin Pract. 2011;119:c214-9.

24. Qunibi WY. Dyslipidemia in Dialysis Patients. Semin Dial. 2015;28:345-53.

25. Mehrotra R, de Boer $\mathbb{I H}$, Himmelfarb J. Adverse effects of systemic glucose absorption with peritoneal dialysis: how good is the evidence? Curr Opin Nephrol Hypertens. 2013;22:663-8.

26. Kronenberg F, Konig P, Neyer U, Auinger M, Pribasnig A, Lang U, Reitinger $J_{\text {, }}$ Pinter G, Utermann G, Dieplinger H. Multicenter study of lipoprotein(a) and apolipoprotein(a) phenotypes in patients with end-stage renal disease treated by hemodialysis or continuous ambulatory peritoneal dialysis. J Am Soc Nephrol. 1995;6:110-20.

27. Vaziri ND, Moradi H. Mechanisms of dyslipidemia of chronic renal failure. Hemodial Int. 2006;10:1-7.

28. Keane WF, Tomassini JE, Neff DR. Lipid abnormalities in patients with chronic kidney disease. Contrib Nephrol. 2011;171:135-42.

29. Pandya V, Rao A, Chaudhary K. Lipid abnormalities in kidney disease and management strategies. World J Nephrol. 2015;4:83-91.

30. Shoji T, Masakane I, Watanabe Y, Iseki K, Tsubakihara Y, Committee of Renal Data Registry JSfDT. Elevated non-high-density lipoprotein cholesterol (non$\mathrm{HDL}-\mathrm{C}$ ) predicts atherosclerotic cardiovascular events in hemodialysis patients. Clin J Am Soc Nephrol. 2011;6:1112-20.

31. Park CH, Kang EW, Park JT, Han SH, Yoo TH, Kang SW, Chang TI. Association of serum lipid levels over time with survival in incident peritoneal dialysis patients. J Clin Lipidol. 2017;11:945-54 e943.

32. Krane V, Winkler K, Drechsler C, Lilienthal J, Marz W, Wanner C, German D, Dialysis Study I. Association of LDL cholesterol and inflammation with cardiovascular events and mortality in hemodialysis patients with type 2 diabetes mellitus. Am J Kidney Dis. 2009;54:902-11.

33. Liu Y, Coresh J, Eustace JA, Longenecker JC, Jaar B, Fink NE, Tracy RP, Powe $\mathrm{NR}$, Klag MJ. Association between cholesterol level and mortality in dialysis patients: role of inflammation and malnutrition. JAMA. 2004:291:451-9.

34. Peev V, Nayer A, Contreras G. Dyslipidemia, malnutrition, inflammation, cardiovascular disease and mortality in chronic kidney disease. Curr Opin Lipidol. 2014;25:54-60.

35. Jankowska M, Cobo G, Lindholm B, Stenvinkel P. Inflammation and proteinenergy wasting in the uremic milieu. Contrib Nephrol. 2017;191:58-71.

\section{Publisher's Note}

Springer Nature remains neutral with regard to jurisdictional claims in published maps and institutional affiliations.

\section{Ready to submit your research? Choose BMC and benefit from:}

- fast, convenient online submission

- thorough peer review by experienced researchers in your field

- rapid publication on acceptance

- support for research data, including large and complex data types

- gold Open Access which fosters wider collaboration and increased citations

- maximum visibility for your research: over $100 \mathrm{M}$ website views per year

At $\mathrm{BMC}$, research is always in progress.

Learn more biomedcentral.com/submissions 